\title{
Autopsy-proven Acute Hemorrhagic Leukoencephalitis in an Elderly Patient
}

\author{
Marlise P. dos Santos, Jason Martin, John Woulfe, Siok P. Lim, Santanu Chakraborty
}

Can J Neurol Sci. 2014; 41: 99-102

Acute Hemorrhagic Leukoencephalitis (AHLE), also known as Weston-Hurst syndrome, or Hurst's disease, is a hyperacute autoimmune demyelinating disease of the white matter, believed to be a severe variant of Acute Disseminated Encephalomyelitis (ADEM). It comprises $2 \%$ of cases of $\mathrm{ADEM}^{1-2}$. Acute Hemorrhagic Leukoencephalitis is relatively rare, with less than 100 cases reported in the scientific literature ${ }^{3}$. We report an autopsy-proven case of AHLE with atypical clinical features and involving primarily the brainstem.

\section{Case Report}

A 75-year-old man presented to the emergency department in September 2007 with acute onset of vertigo, nausea, vomiting. On physical examination he was alert, oriented and afebrile, with a horizontal nystagmus. Approximately eight hours later, bilateral frontal headache and somnolence started. There was no history of recent viral illness, vaccination or travel. His past medical history was significant for hyperlipidaemia, atrial fibrillation, rheumatoid arthritis, gout and hypothyroidism, all on treatment. Previous surgeries included transurethral resection of the prostate in 2006 and coronary artery bypass surgery (x3) in 2004. He quit smoking in 1970 and ingested approximately 5-10 ounces of alcoholic beverage daily. Medications in use for at least four years prior to admission included amiodarone (100-mg OD), irbesartan (75-mg OD), metoprolol (12.5-mg BID), metolazone (2.5-mg OD), furosemide (60-mg OD), levothyroxine (0.2-mg OD), acetylsalicylic acid (81-mg OD), prednisone (5-7.5mg OD), hydroxychloroquine (200-mg BID), risedronic acid (35-mg weekly), rosuvastatin (10-mg PO OD), pantoprazole (40-mg OD), allopurinol (100-mg BID), colchicines (0.6-mg on alternate days), vitamin D (400 IU daily).

There was no leukocytosis in the peripheral blood. Cerebrospinal fluid (CSF) was sterile and demonstrated pleocytosis (90 white blood cells $/ \mathrm{mm}^{3}$, predominantly neutrophils $(30 \%)$, and 101 erythrocytes $/ \mathrm{mm}^{3}$ ), hyperproteinorhachia lacking oligoclonal bands $(1.84 \mathrm{~g} / \mathrm{L})$ and hypoglycorrhachia (CSF:blood glucose $=0.41$ ). The blood cultures were sterile. An unenhanced computed tomography (CT) scan of the head performed on the day of admission was within normal limits.

On day 2 of admission, the drowsiness progressed. The patient rapidly developed dysarthria, right arm paresthesia and progressive respiratory distress, having to be transferred to the intensive care unit. A $1.5 \mathrm{~T}$ magnetic resonance imaging (MRI) study was obtained on this day revealing edema of the medulla oblongata, pons and right middle cerebellar peduncle, with mild contrast enhancement in the medulla oblongata (Figure 1). The gradient-echo sequence was unable to detect haemorrhage.

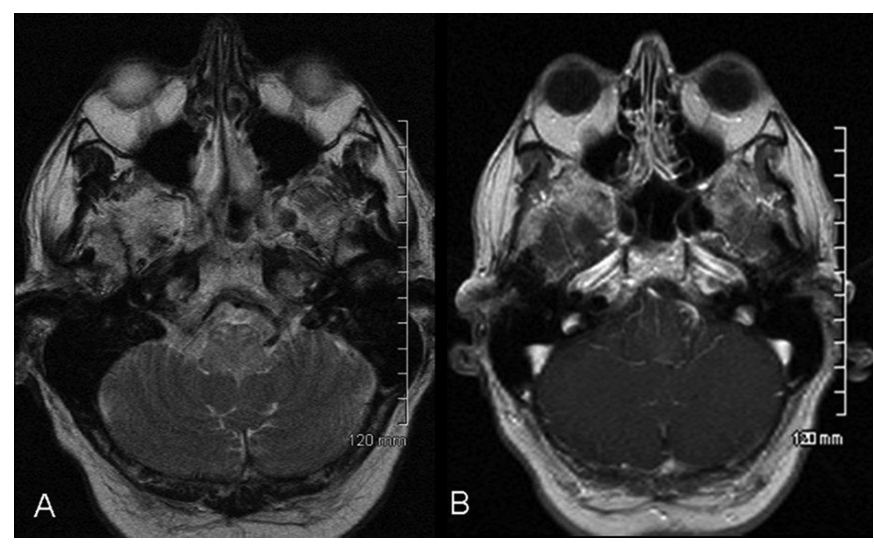

Figure 1: Axial unenhanced T2-weighted fast spin-echo image shows increased signal indicative of edema of the medulla oblongata (A). Axial enhanced T1-weighted fast spin-echo image demonstrates mild contrast enhancement in the medulla oblongata $(B)$.

Diffusion-weighted images showed areas of T2- shine through in the medulla oblongata consistent with vasogenic edema (Figure 2). The differential diagnoses included demyelinating disease (AHLE/ADEM, acute/fulminating multiple sclerosis), vasculitides (Behçet's disease, systemic lupus erythematous) and infection (e.g. rhombencephalitis).

With the provisional diagnosis of viral encephalitis, intravenous antiviral therapy using acyclovir $825-\mathrm{mg}$ q8h was started on day 2, along with prophylactic intravenous cefotaxime (2g q8h), ampicilin ( $2 \mathrm{~g} \mathrm{q} 4 \mathrm{~g}$ ) and vancomycin (1g q12h).

On day 3 , the patient was emergently intubated due to a further rapid decline in level of consciousness with unresponsiveness and a short hypoxic event. A repeat unenhanced CT of the head (not shown) was performed demonstrating mild hydrocephalus and progressive edema involving the brainstem and extending into the thalami and cerebellum. There was no leukocytosis in the peripheral blood.

From the Medical Imaging Department and Ottawa Hospital Research Institute (MPdS, SC), Department of Pathology and Laboratory Medicine and Ottawa Hospital Research Institute (JW), Ottawa, ON, Canada; McMaster University, Hamilton, Ontario Canada (JM); Department of Radiology (SPL), St. Mary's Hospital, London, UK.

Received March 27, 2013. Final Revisions Submitted August 19, 2013.

Correspondence to: Marlise P. dos Santos, Medical Imaging Department, The Ottawa Hospital, 01-1-10A - 1053 Carling Avenue, Ottawa, Ontario, K1Y 4E9, Canada. Email: msantos@toh.on.ca. 


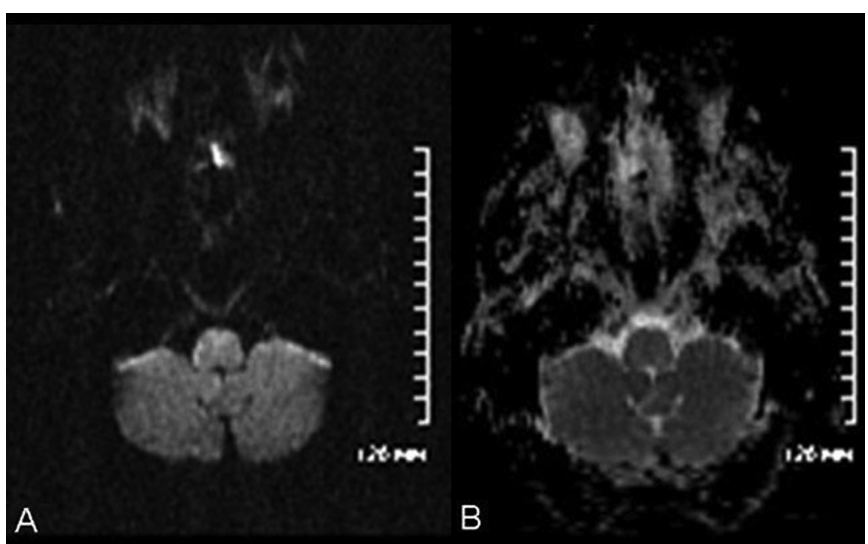

Figure 2: Diffusion-weighted image (A) and apparent diffusion coefficient map (B) both show increased diffusivity in the medulla oblongata indicative of vasogenic edema.

The patient deteriorated further and died later on day 3 of the hospital admission. The repeated blood cultures later on returned negative.

A post-mortem neuropathological examination was performed (Figure 3). On gross external examination of the brain, there was significant swelling, patchy dusky discoloration and softening of the ventral surface of the basis pontis and medulla oblongata (Figure 3D). The cerebellar tonsils were prominent, but not necrotic. There was no brain herniation. On inspection of serial, coronal slices through the cerebral hemispheres, there were well-circumscribed foci of softening and dusky discolouration involving the right and left internal capsules and adjacent lenticular nuclei. Transverse sections through the brainstem showed marked swelling at all levels with multifocal petechial hemorrhages involving the cerebral peduncles and in the basis pontis. The latter showed a larger, well-circumscribed central area of dusky discolouration and parenchymal softening (Figure 3D). Slices of the cerebellum showed swelling with a few petechial hemorrhages identified in the middle cerebellar peduncles bilaterally. Microscopic inspection of the midbrain, pons, medulla and cerebellum revealed a multifocal, angiocentric destructive, inflammatory and demyelinating process consisting of dense perivascular fibrin deposition and neutrophilic infiltrates associated with tissue edema and necrosis with macrophage infiltration (Figure 3A, 3C). Lymphocytes comprised only a minority of the inflammatory cell population. There was mural necrosis and neutrophilic infiltration of venular walls consistent with a necrotizing venulitis. Occasional smaller vessels showed intraluminal organized thrombi. There was relative sparing of arterial vessels. Other vessels showed more acute perivascular hemorrhage with a "ball-and-ring" configuration (Figure 3B). These changes were associated with severe demyelination as revealed in luxol fast-blue (LFB)-stained sections. Macrophages within these areas contained granular LFB-positive material consistent with active demyelination. Similar changes, albeit of somewhat lesser severity, were identified in the basal ganglia (particularly in the globi pallidi), the cerebellar and the cerebral

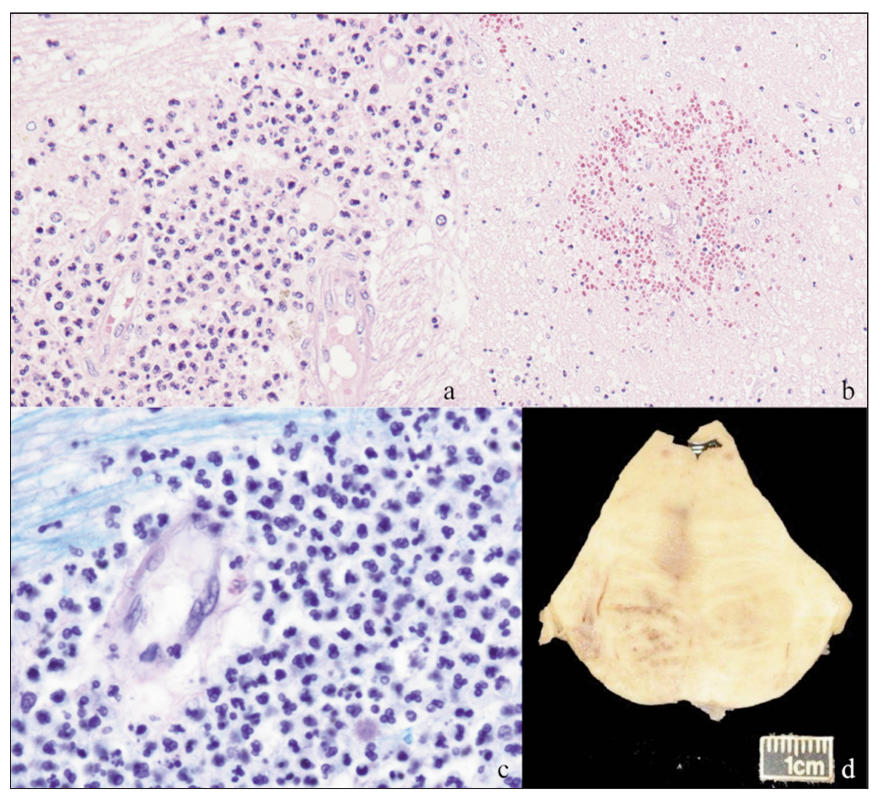

Figure 3: (A) Dense perivascular leukocytic infiltrate in the basis pontis (H and E; X400). (B) More acute perivascular haemorrhage showing a "ball-and-ring' configuration ( $H$ and E; X200). (C) The perivascular inflammatory infiltrates consist predominantly of neutrophils (PAS/LFB; X600). (D) Transverse slice through the pons at the level of the locus coeruleus showing multiple well-circumscribed foci of dusky discolouration in the basis pontis. One of these extends into the inferior pontine tegmentum.

hemispheric white matter. Histological features of viral encephalitis were not present. Special stains revealed no definite bacterial, fungal or acid-fast organisms. Histopathological features of viral encephalitis in the form of perivascular lymphocytic infiltration, microglial proliferation/nodules and nuclear inclusions/neuronophagia were not in evidence. The viral studies on polymerase chain reaction of the brain tissue returned negative. The pathological findings were considered to be consistent with AHLE.

\section{Discussion}

Acute Hemorrhagic Leukoencephalitis was first described in 1941 as a rare form of acute perivenular demyelination and diffuse hemorrhagic necrosis of the central nervous system. It is considered to be the most severe form of $\mathrm{ADEM}^{1-5}$. Patients are usually young with a prodromal illness of upper respiratory tract infection or recent vaccination ${ }^{1,6}$. This is followed by the hyperacute onset of neurologic deficits and death within days. Mortality is high and persistent neurological deficits are common in survivors ${ }^{1}$. Molecular mimicry between antigens of viral or bacterial pathogens and myelin antigens are thought to initiate an autoimmune process in $\mathrm{AHLE}^{1}$ leading to rapid demyelination ${ }^{5-7}$.

Neuroimaging plays an essential role in the early diagnosis of AHLE. Magnetic resonance imaging findings include multifocal asymmetric high signal areas on long repetition time (TR) images within the white matter with or without micro- 
haemorrhage. These lesions are usually confined to the cerebral white matter, particularly in the centrum semiovale, internal capsules, cingulate gyrus, with sparing of the U-fibers ${ }^{4}$. Contrast enhancement of the lesions is variable and depends on the stage and severity of the disease. Diffusivity pattern on diffusionweighted images is also variable ${ }^{4}$. In this case, neuroimaging revealed an atypical non-hemorrhagic acute inflammatory brainstem disease. Micro-hemorrhages could not be detected in the conventional neuroimaging provided (at the time, susceptibility-weighted imaging was not available). The abnormalities spread rapidly into cerebellum and thalami in a 24hour unenhanced follow-up CT study. The imaging differential diagnoses included demyelinating disease (AHLE/ADEM, monophasic or acute multiple sclerosis), vasculitides (Behçet's disease, systemic lupus erythematous) and infection (e.g. rhombencephalitis). There were no MRI features to support suspicion for an acute infarct.

The latency period between the precipitant and the onset of symptoms is shorter in AHLE than in $\mathrm{ADEM}^{3}$. Acute Disseminated Encephalomyelitis and AHLE are both usually preceded by a history of exposure to immunization or infectious illness, typically affecting young patients. Systemic symptoms are more common in AHLE than in ADEM. Cortical signs such as mental status changes and seizures are more relatively common in ADEM. Inflammatory lesions of ADEM may affect any part of the central nervous system, causing a wide spectrum of neurological signs ${ }^{3}$.

Patients with multiple sclerosis, unlike AHLE, typically have a history of relapsing and/or progressive (non-hyperacute) neurological disease, with clinical presentation lacking the encephalopathy features such as respiratory distress, obtundation and drowsiness seen in this case. The progression of aggressive variants of multiple sclerosis (e.g. Schilder's disease, Baló's concentric sclerosis) is usually within a period of months to years and rarely in weeks, and in younger patients. Baló's sclerosis differs from our patient in that there is extensive concentric demyelination ${ }^{8}$. Schilder's myelinoclastic disease is an acute form of demyelination affecting predominantly the centrum ovale $^{9}$. Vasculitides have associated systemic and laboratory findings and usually do not progress as rapidly. The CSF profile and the neuroimaging findings of our patient were inconsistent with brainstem infarction and multiple sclerosis. Rhombencephalitis is usually a particular form of Listerial encephalitis seen in immunocompromised patients. L. monocytogenes is readily cultured from blood in rhomboencephalitis ${ }^{10}$. Our patient was not immunocompromised. His blood cultures returned sterile.

This case primarily underlines the complexity of differentiating AHLE from ADEM. This patient was not in the typical age group of presentation of either ADEM or AHLE. There was absence of a prodromal illness, an important clue in making the diagnosis of ADEM. On neuroimaging, the lesions in ADEM compared to AHLE are smaller, have less edema or mass effect, and rarely show hemorrhage. The brainstem was abnormal in this case and there was no obvious haemorrhage in the MRI sequences provided. Pleocytosis of CSF in ADEM is predominantly lymphocytic, and predominantly polymorphonuclear in AHLE, like in this case. Hyperproteinorhachia, normal glucose level, raised $\operatorname{IgG}$ and presence of myelin basic protein ${ }^{1}$ may also be seen in AHLE. In our case, oligoclonal bands were not observed in the CSF. Acute Hemorrhagic Leukoencephalitis is also commonly associated with peripheral leucocytosis ${ }^{1-4}$, not evident in our patient likely due to poor reactive response related to his age and chronic use of prednisone. The pathologic features of ADEM include perivascular infiltrates and consist predominantly of lymphocytes and macrophages with relatively few neutrophils and absence of perivascular haemorrhage or necrosis of the venules ${ }^{1}$. Acute Hemorrhagic Leukoencephalitis shows a neutrophilic infiltrate with perivascular hemorrhage and the distinctive feature is a necrotising vasculitis of the venules ${ }^{1}$. The neuropathologic features found in autopsy were typical for AHLE.

Also challenging was the differentiation between AHLE and Marburg's acute monophasic multiple sclerosis, which in contrast to AHLE invariably shows less rapid presentation. It also demonstrates hystopathological findings of giant astrocytes, sharp-edge demyelinated lesions, and relative axonal preservation, none of which were seen in this case. The CSF study usually reveals a moderate increase of protein content and cells but, in contrast to our case, oligoclonal bands are invariably present ${ }^{11}$.

The treatment of ADEM and AHLE does not differ significantly. Given the probable etiology of AHLE, successful management requires early diagnostic suspicion, aggressive management of cerebral edema, and immunosuppression. In this case, the rapid and fulminating progression of the disease led to patient mortality without a final diagnosis, highlighting the importance of having a low threshold in considering AHLE in rapidly progressive white matter disease ${ }^{3}$. Methylprednisone is routinely given (1g IV daily, three to five days). If the patient does not respond to this treatment, intravenous immunoglobulin may be administered ${ }^{3}$. A few reports of the use of therapeutic plasma exchange have demonstrated survival with minimal neurological impartment. ${ }^{12-14}$

In conclusion, although AHLE is rare, its high mortality and morbidity makes prompt diagnosis essential. One should have a low threshold in considering this diagnosis particularly in a rapidly progressive white matter disease. Aggressive management of cerebral edema, immunomodulation therapy and therapeutic plasma exchange have been reported to increase survival with improved neurologic outcome.

\section{ACKNOWLEDGMENT}

The authors thank Betty Anne Schwarz (Medical Imaging Department Research Manager) and Christina Tsoukanas (Medical Imaging Department Research Assistant) for their relentless support of our research endeavours including this manuscript.

\section{REFERENCES}

1. Leake JA, Albani S, Kao AS, et al. Acute disseminated encephalomyelitis in childhood: epidemiologic, clinical and laboratory features. Pediatr Infect Dis J. 2004;23(8):756-64.

2. Tenembaum S, Chamoles N, Fejerman N. Acute disseminated encephalomyelitis: a long-term follow-up study of 84 pediatric patients. Neurology. 2002;59:1224-31.

3. Davies NW, Sharief MK, Howard RS. Infection-associated encephalopathies - their investigation, diagnosis, and treatment. J Neurol. 2006;253(7):833-45. 
4. Gibbs WN, Kreidie MA, Kim RC, et al. Acute hemorrhagic leukoencephalitis: neuroimaging features and neuropathologic diagnosis. J Comput Assist Tomogr. 2005;29:689-93.

5. Lann MA, Lovell MA, Kleinschmidt-DeMasters BK. Acute hemorrhagic leukoencephalitis: a critical entity for forensic pathologists to recognize. Am J Forensic Med Pathol. 2010;31 (1):7-11.

6. Pinto PS, Taipa R, Moreira B, et al. Acute hemorrhagic leukoenphalitis with severe brainstem and spinal cord involvement: MRI features with neuropathological confirmation. J Magn Reson Imaging. 2011;33(4):957-61 .

7. Dale RC. Acute disseminated encephalomyelitis. Semin Pediatr Infect Dis. 2003;14:90-5.

8. Karaarslan E, Altintas A, Senol U, et al. Baló's Concentric Sclerosis: clinical and radiologic features of five cases. Am J Neuroral. 2001;22:1362-7.

9. Kotil K, Kalayci M, Koseoglu T, et al. Myelinoclastic diffuse sclerosis (Schilder's disease): report of a case and review of the literature. Br J Neurosug. 2002;16:516-19.
10. Miller DH, Weinshernker BG, Filippi M, et al. Differential diagnosis of suspected multiple sclerosis: a consensus approach. Multiple Sclerosis. 2008;14:1157-74.

11. Mendez MF, Pogacar S. Malignant monophasic multiple sclerosis or "Marburg's disease". Neurology. 1988;38(7):1153.

12. Seales D, Greer M. Acute hemorrhagic leukoencephalitis: a successful recovery. Arch Neurol 1991;48:1086-8.

13. Markus R, Brew BJ, Turner J, et al. Successful outcome with aggressive treatment of acute hemorrhagic leukoencephalitis. J Neurol Neurosurg Psychiatry. 1997;63:551-2.

14. Ryan LJ, Bowman R, Zantek ND, et al. Use of therapeutic plasma exchange in the management of acute hemorrhagic leukoencephalitis: a case report and review of the literature. Transfusion. 2007;6:981-6. 